\title{
Allergy Immunology
}

A391C polymorphism 111

Ac2-26 137

A/J mouse 264

Adeno-associated virus 207

Adjuvant 336

Adolescent 42

Affinity purification 33

Airway inflammation 25

- remodeling 207

Allergen exposure 246

- extracts 295

- isoforms 295

Allergenicity 195

Allergens 345

Allergic conjunctivitis 349

- disease 310

- disorder 249

- rhinitis 246, 295, 336

Allergist 57

Allergy 227

Allerwatch ${ }^{\circledR} 349$

Amb a 1164

Anaphylaxis 318

Angioedema 81

Annexin 1137

Antibiotics 57

Antidepressants 63

Antigen presentation 89

Art v 1164

- $\quad 3164$

Asc 13195

Ascaris lumbricoides 195

Asthma 25, 42, 63, 69, 137, 195, 207, 249, 286

Atopic 318

- dermatitis 49, 236, 353

Atopy 42

Autosomal recessive 76

Bahia grass pollen 295

Belgium 173

Berinert $^{\circledR}$ P 356

Bet v1 278

- v2 278

- v4 specific IgE 278

Biogenics 236

Birch pollen 278

Bronchial hyperresponsiveness 42

C1 esterase inhibitor deficiency 81

- inhibitor concentrate 81

- _ deficiency 356
Case-control study 328

CCL2 185

CD203c 318

Cellular responses 128

Child Behavior Checklist 249

Children 236, 249

Chimeric IgE antibody 33

Chronic hypersensitivity pneumonitis 264

- rhinitis 155

Cockroach allergen 318

Coefficient of inbreeding 76

Conalbumin 33

Conjunctival allergen challenge 149

Consanguinity 76

Contact urticaria 345

Cow's milk infant formula 310

Cross-reactive carbohydrate determinants 164

Cytokine(s) 185, 227

Dansyl 119

Dermatophagoides 69

Dog allergens 258

- allergy diagnosis 258

Eczema 328

Edema 286

Egg allergy 33

Eosinophil-derived neurotoxin 119

Eosinophil granule proteins 119

- recruitment 137

Eosinophils 119, 286

Epicutaneous immunotherapy 299

Epidemiology 76

Exhaled breath condensate 25

Fibrogenesis 264

Filaggrin 353

Flow cytometry 318

Food allergy 6, 299, 345

- -, diagnosis 216

- challenge 216

Fungi-specific IgE 49

Gene 353

- therapy 207

Genetic defects 76

Grass pollen 336

- _ allergens 295

Hereditary angioedema 81, 356

Histamine reactivity 63
Homology structure model 111

Human basophils 318

Humoral responses 128

Hymenoptera allergy 353

$\operatorname{IgE} 6,15,195$

- reactivity 295

- receptor 15

IL-6 227

ImmunoCAP 33

Immunochromatography 349

Immunoglobulin E 177

Immunoglobulins 119

-, intravenous 173

Inflammation 42, 286

Interleukin-5 207

Interleukin-13 328

Isomaltitol 286

Japanese children 328

$\beta$-Lactam antibiotic 57

Lactobacillus acidophilus L-92 236

Latex allergy 149

Leukotrienes 227

Lipid rafts 89

- transfer protein 345

Long-term replacement treatment 81

Lysozyme 33

Mast cell 177

Mite allergy 195

Monophosphoryl lipid A 336

Morbidity 76

Mucociliary clearance 286

Mugwort pollen 164

Neutrophils 286

Off-label use 173

Omalizumab 25, 177, 318

Ovalbumin 33

Overweight 42

Ovomucoid 33

Par j 2164

Partial protein-hydrolyzed infant formula 310

Pas n 1295

Pasteurized C1 inhibitor concentrate 356

Pattern recognition receptors 227

Peach 345 
Peanut 299

- allergy 216, 318

Penicillin 57

- allergy 57

Peptidoglycan 227

Pharmacist 57

Plethysmography 299

Polcalcin 164

Pollen allergy 246

- cytoplasmic granules 128

- season 246

Polymorphism 328

Population survey 155

Prevalence 155

Primary immunodeficiency 76

Probiotics 236

Profilin 164

Prostaglandin $\mathrm{D}_{2} 137$

Pseudotyping 89

Psychosocial adaptation 249

RANTES 25

Receptor processing 15
Recombinant allergens 216, 278

Red blood cell binding 111

Registry 76

Reimbursement 173

Resistance-compliance 299

Respiratory allergic disease 49

- allergy 6

- syncytial virus 185

Rhinitis 63, 69

Risk factors 155

Sal k 1164

Selective serotonin reuptake inhibitors 63

Sensitization 6, 249, 310

Sephadex 286

Serum interleukin 9246

Siglec-9 111

Skin allergy 6

- prick test 63

- _ - extracts 258

Sublingual immunotherapy 69, 336

Superoxides 119
T cell receptor-mediated signaling 111

Tear 349

Th1 185

Th2 185, 264

Timothy grass pollen 128

Total IgE 349

Tree nut allergy 318

Tricyclic antidepressants 63

Tropomyosin 195

Tumor necrosis factor 286

Ultra-rush 69

Urticaria 177

Viaskin ${ }^{\circledR} 299$

Virus-like particles 89

Visual Analog Scale 63 